\title{
NET SPACES AND BOUNDEDNESS OF INTEGRAL OPERATORS
}

\author{
ERLAN NURSULTANOV AND SERGEY TIKHONOV
}

ABSTRACT. In this paper we introduce new functional spaces which we call the net spaces. Using their properties, the necessary and sufficient conditions for the integral operators to be of strong or weaktype are obtained. The estimates of the norm of the convolution operator in weighted Lebesgue spaces are presented.

\section{INTRODUCTION}

Let $(\Omega, \mathfrak{F}, \mu)$ be a measurable space, where $\mu$ is a $\sigma$-additive measure, $\mathfrak{F}$ is the algebra of the measurable sets with identity $\Omega$. The space $L_{p}(\Omega, \mu)$ is the collection of all those measurable functions $f$ satisfying

$$
\|f\|_{L_{p}(\Omega, \mu)}=\left(\int_{\Omega}|f(x)|^{p} d \mu\right)^{\frac{1}{p}}<\infty .
$$

The distribution of a measurable function $f$ on $\Omega$ is defined by

$$
m(\sigma, f)=\mu\{x \in \Omega:|f(x)|>\sigma\} .
$$

Then $f^{*}(t)=\inf \{\sigma: m(\sigma, f) \leqslant t\}$ is the decreasing rearrangement of $f$.

Let $0<p \leqslant \infty$ and $0<q \leqslant \infty$. The Lorentz space $L_{p q}(\Omega, \mu)$ is defined [BS, Ch. 4] by those measurable functions $f$ such that

$$
\|f\|_{L_{p q}}=\left(\int_{0}^{\infty}\left(t^{1 / p} f^{*}(t)\right)^{q} \frac{d t}{t}\right)^{1 / q}<\infty
$$

when $0<q<\infty$, and

$$
\|f\|_{L_{p \infty}}=\sup _{t} t^{1 / p} f^{*}(t)<\infty
$$

when $q=\infty$. We also define

$$
f^{* *}(x)=\frac{1}{x} \int_{0}^{x} f^{*}(t) d t
$$

2000 Mathematics Subject Classification. Primary 46E30; Secondary 47G10, 45P05.

Key words and phrases. Net spaces, Lorentz spaces, Integral Operators, Strong and Weak-type Boundedness, Convolution. 
In this paper we study the weak and strong $(p, q)$-boundedness of the integral operator

$$
T f(y)=\int_{D} K(x, y) f(x) d \nu_{x}
$$

in weighted Lebesgue and Lorentz spaces. Here the kernel $K(x, y)$ is a $\nu \otimes \mu$-measurable locally integrable function on $D \times \Omega$.

The boundedness of the Hardy-type transforms, the potential operators, the Laplace and Fourier transforms, the Riemann-Liouville operators in the weighted spaces have been extensively studied; and in many cases the complete answers were presented as criteria in terms of weights (see the texts $[\mathrm{CRS}],[\mathrm{KP}],[\mathrm{EKM}]$ and the earlier papers $[\mathrm{AH}],[\mathrm{AS}],[\mathrm{BH} 1],[\mathrm{BH} 2]$, $[\mathrm{CF}],[\mathrm{Mu}])$.

In the general case, from the results by Kantorovič-Vulih and DunfordPettis ([DP], [KV], [KA, Ch. 11,§1]), it follows that for $1 \leqslant p<\infty$ and $1<q \leqslant \infty$

$$
\begin{aligned}
\|T\|_{L_{p}(D, \nu) \rightarrow L_{\infty}(\Omega, \mu)} & =e s s \sup _{y \in \Omega}\|K(\cdot, y)\|_{L_{p^{\prime}}(D, \nu)}, \\
\|T\|_{L_{1}(D, \nu) \rightarrow L_{q}(\Omega, \mu)} & =e s s \sup _{x \in D}\|K(x, \cdot)\|_{L_{q}(\Omega, \mu)} .
\end{aligned}
$$

Necessary and sufficient conditions for $T$ to be bounded from $L_{p}(D, \nu)$ to $L_{q}(\Omega, \mu)$ can be obtained using the factorization technique ([Ch], [Ga], [GR], [H1], [H2], [Ja]). In particular, such results were given for $1<q<p<\infty$; $1 \leqslant p \leqslant \infty, q=1 ;$ and $p=\infty, 1 \leqslant q \leqslant \infty$.

We also recall the results on the boundedness of the convolution-type operator in the Lorentz spaces (Hölder-type inequalities): we refer to O'Neil [ON], Yap [Ya], and Blozinski [Bl]. In particular, the following Young-O'Neil inequality is known

$$
\|A f\|_{L_{q, s}(\Omega, d x)} \leqslant C\|K\|_{L_{r, t_{1}}(\Omega-D, d x)}\|f\|_{L_{p, t_{2}}(D, d x)},
$$

where $1<p, q, r<\infty, 1+\frac{1}{q}=\frac{1}{p}+\frac{1}{r}, \frac{1}{t_{1}}+\frac{1}{t_{2}} \geqslant \frac{1}{s}$,

$$
A f(y)=\int_{D} K(x-y) f(x) d x,
$$

and $d \mu=d \nu=d x$ are the $n$-dimensional Lebesgue measures. In particular, we have

$$
\|A\|_{L_{p}(D, d x) \rightarrow L_{q}(\Omega, d x)} \leqslant C\|K\|_{L_{r, \infty}(\Omega-D, d x)} .
$$

However, in the case of non-homogeneous measures, operator (1.5) does not satisfy all requirements from $[\mathrm{ON}]$ and needs thorough investigation. For the power weights the Young-O'Neil inequality was generalized by Kerman [Ke]. 
By $C, C_{i}, c$ we will denote positive constants that may be different on different occasions. Also, $F \asymp G$ means that $F \leqslant C G$ and $G \leqslant C F$.

This paper is organized as follows. In section 2 we define the new function spaces, which we call the net spaces $N_{p q}$ and which are natural generalizations of the Lorentz spaces. It turns out that they have similar embedding and interpolation properties as the Lorentz spaces. Particularly, we have

$$
\cdots \hookrightarrow N_{p 1} \hookrightarrow \cdots \hookrightarrow N_{p p} \hookrightarrow \cdots \hookrightarrow N_{p q} \hookrightarrow \cdots \hookrightarrow N_{p \infty}, \quad 1 \leqslant p \leqslant q .
$$

Section 3 consists the general results on the boundedness of operator (1.1). The necessary and sufficient conditions for $(1.1)$ to be of $(p, q)$ quasi-weak and $(p, q)$ weak-type are presented. We also give a sufficient condition for (1.1) to be bounded in the Lorentz spaces. We finish with Sections 4 and 5 which deals with the convolution operator in $L_{p}$ and Lorentz spaces respectively. Two important cases: of regular kernel and regular measures and investigated. In particular, Theorems 4.1 and 5.1 imply Kerman's results.

\section{The Net SPACE}

First, let $(\Omega, \mathfrak{F}, \mu)$ be the measurable space and let $M^{*}$ be the collection of all $\mu$-measurable sets of positive measure, i.e.,

$$
M^{*}:=\{e \in \mathfrak{F}: 0<\mu(e)<\infty\} .
$$

Then we will call the net $M$ a fix subset from $M^{*}$.

Let $f(x)$ be a measurable function on $\Omega$ such that $f(x)$ is integrable on any $e \in M$. We define the average function $\bar{f}(t, M)$ on $t \in(0, \infty)$ as follows

$$
\bar{f}(t, M):=\bar{f}(t, M, \mu)=\sup \frac{1}{\mu(e)}\left|\int_{e} f(x) d \mu\right|,
$$

where the supremum is taken over all sets $e \in M$ such that $\mu(e)>t$, $t \in(0, \infty)$. In the case of $\sup _{e \in M} \mu(e)=\alpha<\infty$ we set $\bar{f}(t, M)=0$ for $t>\alpha$.

We say that a $\mu$-measurable function $f(x)$ belongs to the Net space $N_{p, q}(M, \mu), 0<p, q \leqslant \infty$, if

$$
\|f\|_{N_{p q}(M, \mu)}=\left(\int_{0}^{\infty}\left(t^{1 / p} \bar{f}(t, M)\right)^{q} \frac{d t}{t}\right)^{\frac{1}{q}}<\infty
$$

for $q<\infty$ and

$$
\|f\|_{N_{p \infty}(M, \mu)}=\sup _{e \in M} \frac{1}{(\mu(e))^{1 / p^{\prime}}}\left|\int_{e} f(x) d \mu\right|<\infty
$$

for $q=\infty$. Here and subsequently $p^{\prime}=p /(p-1)$. 


\section{Properties.}

(1) If $M_{1} \subset M_{2}$, then $N_{p q}\left(M_{2}\right) \hookrightarrow N_{p q}\left(M_{1}\right)$.

(2) For $0<q \leqslant q_{1} \leqslant \infty$ we have $N_{p q}(M, \mu) \hookrightarrow N_{p q_{1}}(M, \mu)$.

(3) If the net $M$ is such that $\sup _{e \in M} \mu e=\alpha<\infty$, then for $0<p<p_{1} \leqslant \infty$ and $0<q, q_{1} \leqslant \infty$ we have $N_{p_{1} q_{1}}(M, \mu) \hookrightarrow N_{p q}(M, \mu)$.

(4) The net space $N_{p, q}(M, \mu)$ is a quasi-normable space as the factorspace over the kernel $\left\{f: \int_{e} f(x) d \mu=0, e \in M\right\}$. In the case of $q \geqslant 1, N_{p, q}(M, \mu)$ is a normable space.

Proof. Items (1)-(4) follow from the definition of the net space and a similar argument which we use to prove corresponding properties for the Lorentz spaces.

Example. (1) Let us consider two nets: $M_{0}$ and $M_{1}$ such that $M_{0}=$ $\{[a, b] \subset \mathbb{R}: a<b\} \subset M_{1}=M^{*}$. Then the following inequalities are true (for the proof see Example 3.1 in the next section):

$$
C_{1}\|f\|_{N_{p q}\left(M_{0}, d x\right)} \leqslant\|\hat{f}\|_{L_{p^{\prime} q}(\mathbb{R}, d x)} \leqslant C_{2}\|f\|_{N_{p q}\left(M_{1}, d x\right)}, \quad 1<p \leqslant 2
$$

where $\hat{f}$ is the Fourier transform of $f$. This example shows that choosing the appropriate nets, one can "catch" the quantity in which we are interested. We also note that $N_{p, q}(M, \mu)$ was introduced in $[\mathrm{Nu}]$ for the case when $\mu$ is $n$-dimensional Lebesgue measure.

(2) Let $M=\{[a, b] \subset \mathbb{R}: a<b\}$ and let $1<p<\infty, 0<q \leqslant \infty$. The function $f(x)=(-1)^{[x]}$ ([x] is an integer part of $x$ ) belongs to $N_{p, q}(M, \mu)$ but $|f|$ does not belong to $N_{p, q}(M, \mu)$.

Indeed, if $\{t\}$ is the fractional part of $t$, i.e., $\{t\}=t-[t]$, then

$$
\bar{f}(t, M)= \begin{cases}\frac{\max (\{t\}, 1-\{t\})}{t} \leqslant \frac{1}{t}, & t \geqslant 1 \\ 1, & t \leqslant 1 .\end{cases}
$$

Therefore,

$$
\|f\|_{N_{p q}(M, \mu)} \leqslant\left(\int_{0}^{1} t^{\frac{q}{p}-1} d t+2 \int_{1}^{\infty} t^{\left(\frac{1}{p}-1\right) q-1} d t\right)^{1 / q}<\infty .
$$

Thus, generally speaking, the net space is not a lattice [BS, p. 6].

(3) Let $M=\{[a, b]: \mu([a, b])=1\}$. Then for any $0<p, q \leqslant \infty$ we have

$$
N_{p q}(M, \mu) \equiv\left\{\mu \text {-measurable } f: \sup _{k}\left|\int_{k}^{k+1} f(x) d \mu\right|<\infty\right\} .
$$


(4) Let $1<p<\infty, 0<q \leqslant \infty, M=\{[0, a]: a>0\}$, and the function $f_{\Omega}(x)$ is characteristic function of the set $\Omega=\bigcup_{k=1}^{\infty}\left[a_{k}, a_{k}+1\right]$. If $a_{k}=\left(k \ln ^{1 / q} k\right)^{p^{\prime}}$, then $f_{\Omega} \in N_{p, q+\varepsilon}(M)$ for any $\varepsilon>0$ but $f_{\Omega} \notin$ $N_{p, q}(M)$. Therefore, the space $N_{p, q}(M, \mu)$ is sensible to regard the distribution of the oscillation of functions.

(5) Let $1<p<\infty, 0<q \leqslant \infty$, and $M=\left\{\left[\sum_{k=1}^{n} k, \sum_{k=1}^{n+1} k\right]: n \in \mathbb{N}\right\}$. Then the net space $N_{p q}(M, \mu)$ is isomorphic to the space

$$
\left\{a=\left\{a_{n}\right\}_{n=1}^{\infty}: \sum_{k=1}^{\infty}\left(k^{1 / p} \sup _{n \geqslant k}\left|a_{n}\right|\right)^{q} \frac{1}{k}<\infty\right\} .
$$

(6) Let $1<p<\infty, 0<q \leqslant \infty$, and $M=\{[0, n]: n \in \mathbb{N}\}$. Then the net space $N_{p q}(M, \mu)$ is isomorphic to the space

$$
\left\{a=\left\{a_{n}\right\}_{n=1}^{\infty}: \sum_{k=1}^{\infty}\left(k^{1 / p} \sup _{n \geqslant k} \frac{1}{n}\left|\sum_{i=1}^{n} a_{i}\right|\right)^{q} \frac{1}{k}<\infty\right\} .
$$

(7) Suppose that $1<p \leqslant \infty$ and $0<q \leqslant \infty$; then $N_{p, q}\left(M^{*}, \mu\right) \equiv$ $L_{p, q}(\Omega, \mu)$. This follows from the fact that

$$
\|f\|_{L_{p, q}(\Omega)} \asymp \begin{cases}\left(\int_{0}^{\infty}\left(t^{1 / p} f^{* *}(t)\right)^{q} \frac{d t}{t}\right)^{1 / q}, & 1<p<\infty, q<\infty ; \\ \sup _{t>0} t^{1 / p} f^{* *}(t), & 1<p \leqslant \infty, q=\infty\end{cases}
$$

and Lemma 2.1 below. For the case of $0<p, q \leqslant \infty$, see [EO]. We also note that $N_{1 q}(M, \mu) \neq L_{1 q}(D, \mu)$ but $N_{1 \infty}(M, \mu)=L_{1}(D, \mu)$.

We will need the following lemma.

Lemma 2.1. Let $f$ be a measurable and integrable on the elements of the net $M^{*}$. Then we have

$$
\bar{f}\left(t, M^{*}\right) \leqslant f^{* *}(t) \leqslant 4 \bar{f}\left(t / 3, M^{*}\right), \quad t>0
$$

Proof. Let $t \in(0, \infty)$ and for any $e \in M^{*}$ such that $\mu(e)=t$ and for a function $f$ we define the following sets $\omega_{1}=\{x \in e: f(x) \geqslant 0\}$ and $\omega_{2}=\{x \in e: f(x)<0\}$. Then

$$
\begin{aligned}
\int_{e}|f(x)| d \mu & =\int_{\omega_{1}} f(x) d \mu-\int_{\omega_{2}} f(x) d \mu \\
& \leqslant 2 \max \left\{\left|\int_{\omega_{1}} f(x) d \mu\right|,\left|\int_{\omega_{2}} f(x) d \mu\right|\right\} .
\end{aligned}
$$


6

ERLAN NURSULTANOV AND SERGEY TIKHONOV

We can assume that

$$
\left|\int_{\omega_{1}} f(x) d \mu\right| \geqslant\left|\int_{\omega_{2}} f(x) d \mu\right| .
$$

We consider two cases: 1). $\left.\mu\left(\omega_{1}\right) \geqslant \frac{1}{2} \mu\left(\omega_{2}\right) ; 2\right) . \quad \mu\left(\omega_{1}\right)<\frac{1}{2} \mu\left(\omega_{2}\right)$. In case 1),

$$
\mu\left(\omega_{1}\right) \geqslant \frac{1}{2} \mu\left(\omega_{2}\right) \quad \text { implies } \quad \mu\left(\omega_{1}\right) \geqslant \frac{\mu(e)}{3}=\frac{t}{3}
$$

and

$$
\begin{aligned}
\frac{1}{\mu(e)} \int_{e}|f(x)| d \mu & \leqslant \frac{2}{\mu(e)}\left|\int_{\omega_{1}} f(x) d \mu\right| \\
& \leqslant \frac{2}{\mu\left(\omega_{1}\right)}\left|\int_{\omega_{1}} f(x) d \mu\right| \leqslant 2 \bar{f}\left(\frac{t}{3}, M^{*}\right) .
\end{aligned}
$$

In case 2$)$, we have $2 \mu\left(\omega_{1}\right)<\mu\left(\omega_{2}\right)$, i.e., $\mu\left(\omega_{2}\right)>2 \mu(e) / 3=2 t / 3$. Then there exist $\omega_{2}^{1}$ and $\omega_{2}^{2}$ from $M^{*}$ such that $\mu\left(\omega_{2}^{1} \bigcap \omega_{2}^{2}\right)=0, \omega_{2}^{1} \bigcup \omega_{2}^{2}=\omega_{2}$, and $\mu\left(\omega_{2}^{i}\right)=\mu\left(\omega_{2}\right) / 2>t / 3$.

Further, by definition of the set $\omega_{2}$, we have

$$
\begin{aligned}
\left|\int_{\omega_{1}} f(x) d \mu\right| & \geqslant\left|\int_{\omega_{2}} f(x) d \mu\right|=\left|\int_{\omega_{2}^{1}} f(x) d \mu\right|+\left|\int_{\omega_{2}^{2}} f(x) d \mu\right| \\
& \geqslant 2 \min \left(\left|\int_{\omega_{2}^{1}} f(x) d \mu\right|,\left|\int_{\omega_{2}^{2}} f(x) d \mu\right|\right)=2\left|\int_{\omega_{2}^{i_{0}}} f(x) d \mu\right| .
\end{aligned}
$$

Here, $\omega_{2}^{i_{0}}$ are sets where the infimum is attained.

Let now $\omega=\omega_{1} \bigcup \omega_{2}^{i_{0}}$, then $|\omega|>|e| / 3$ and

$$
\begin{aligned}
\left|\int_{\omega} f(x) d \mu\right| & =\left|\int_{\omega_{1}} f(x) d \mu+\int_{\omega_{2}^{i_{0}}} f(x) d \mu\right| \\
& \geqslant\left|\int_{\omega_{1}} f(x) d \mu\right|-\left|\int_{\omega_{2}^{i_{0}}} f(x) d \mu\right| \geqslant \frac{1}{2}\left|\int_{\omega_{1}} f(x) d \mu\right| .
\end{aligned}
$$

Hence,

$$
\begin{aligned}
\frac{1}{\mu(e)} \int_{e}|f(x)| d \mu & \leqslant \frac{2}{\mu(e)}\left|\int_{\omega_{1}} f(x) d \mu\right| \\
& \leqslant \frac{4}{\mu(\omega)}\left|\int_{\omega} f(x) d \mu\right| \leqslant 4 \bar{f}\left(t / 3, M^{*}\right) .
\end{aligned}
$$

Collecting this,

$$
f^{* *}(t)=\sup _{\mu(e)=t} \frac{1}{\mu(e)} \int_{e}|f(x)| d \mu
$$


and (2.2), we arrive at the right hand side estimate in (2.1). Let us prove the left hand side estimate.

$$
\begin{aligned}
\bar{f}\left(t, M^{*}\right) & =\sup _{\mu(e) \geqslant t} \frac{1}{\mu(e)}\left|\int_{e} f(x) d \mu\right| \\
& \leqslant \sup _{\mu(e) \geqslant t} \frac{1}{\mu(e)} \int_{e}|f(x)| d \mu \\
& =\sup _{\mu(e) \geqslant t} \frac{1}{\mu(e)} \int_{0}^{\mu(e)} f^{*}(s) d s \\
& =\sup _{\mu(e) \geqslant t} \frac{1}{\mu(e)}\left(\int_{0}^{t} f^{*}(s) d s+\int_{t}^{\mu(e)} f^{*}(s) d s\right) \\
& \leqslant \sup _{\mu(e) \geqslant t} \frac{1}{\mu(e)}\left(\int_{0}^{t} f^{*}(s) d s+(\mu(e)-t) \frac{1}{t} \int_{0}^{t} f^{*}(s) d s\right) \\
& =\frac{1}{t} \int_{0}^{t} f^{*}(s) d s=f^{* *}(t) .
\end{aligned}
$$

The proof is now complete.

Interpolation. We next discuss the interpolation of the net spaces using the real method of Peetre [BS, Ch. 5]. If $X_{0}$ and $X_{1}$ are a pair of quasinormed spaces which are continuously embedded in a linear Hausdorff space $X$, i.e., $\left(X_{0}, X_{1}\right)$ is a compatible couple, their $K$-functional is defined for any $f \in X_{0}+X_{1}$ by

$$
K(f, t):=K\left(f, t ; X_{0} ; X_{1}\right):=\inf _{f=f_{0}+f_{1}}\left\|f_{0}\right\|_{X_{0}}+t\left\|f_{1}\right\|_{X_{1}} .
$$

For each $0<\theta<1,0<q \leqslant \infty$, the space $X_{\theta, q}:=\left(X_{0} ; X_{1}\right)_{\theta, q}$ is the collection of all functions $f \in X_{0}+X_{1}$ for which

$$
\|f\|_{\theta, q}:=\left(\int_{0}^{\infty}\left(t^{-\theta} K(f, t)\right)^{q} \frac{d t}{t}\right)^{\frac{1}{q}}
$$

is finite (with the usual adjustment on the right side of $(2.3)$ when $q=\infty$ ). This is an interpolation space since it follows easily from the definition of the $K$-functional that each linear operator which is bounded on $X_{0}$ and $X_{1}$ is also bounded on $X_{\theta, q}$.

We are interested in interpolation for a pair of net spaces.

Theorem 2.1. Let $0<p_{0}<p_{1} \leqslant \infty, 0<q_{0}, q_{1}, q \leqslant \infty$, and let $M$ be any net in $\mathfrak{F}$. We have

$$
\left(N_{p_{0}, q_{0}}(M, \mu), N_{p_{1}, q_{1}}(M, \mu)\right)_{\theta, q} \hookrightarrow N_{p, q}(M, \mu),
$$

where $0<\theta<1$ and $1 / p=(1-\theta) / p_{0}+\theta / p_{1}$. 
Proof. Because of the embedding $N_{p, q}(M, \mu) \hookrightarrow N_{p, \infty}(M, \mu)$ it is sufficient to show

$$
\left(N_{p_{0}, \infty}(M, \mu), N_{p_{1}, \infty}(M, \mu)\right)_{\theta, q} \hookrightarrow N_{p, q}(M, \mu) .
$$

Let $f=f_{0}+f_{1}$, where $f_{0} \in N_{p_{0}, \infty}(M, \mu)$ and $f_{1} \in N_{p_{1}, \infty}(M, \mu)$. Clearly

$$
\bar{f}(t, M) \leqslant \bar{f}_{0}(t, M)+\bar{f}_{1}(t, M)
$$

and then denoting $v(t)=t^{\frac{p_{0} p_{1}}{p_{1}-p_{0}}}$ we have

$$
\begin{aligned}
\sup _{v(t) \geqslant s>0} s^{1 / p_{0}} \bar{f}(s, M) & \leqslant \sup _{s>0} s^{1 / p_{0}} \bar{f}_{0}(s, M)+\sup _{v(t)>s} s^{\frac{1}{p_{0}}-\frac{1}{p_{1}}+\frac{1}{p_{1}}} \bar{f}_{1}(s, M) \\
& \leqslant \sup _{s>0} s^{1 / p_{0}} \bar{f}_{0}(s, M)+t \sup _{s>0} s^{1 / p_{1}} \bar{f}_{1}(s, M) .
\end{aligned}
$$

Further, taking the infimum over all $f_{0}$ and $f_{1}$, such that $f=f_{0}+f_{1}$, we get

$$
\sup _{v(t) \geqslant s>0} s^{1 / p_{0}} \bar{f}(s) \leqslant K\left(t, f ; N_{p_{0} \infty}, N_{p_{1} \infty}\right) .
$$

Thus for $0<q \leqslant \infty$

$$
\begin{gathered}
\left(\int_{0}^{\infty}\left(t^{-\theta} K(t, f)\right)^{q} \frac{d t}{t}\right)^{\frac{1}{q}} \geqslant\left(\int_{0}^{\infty}\left(t^{-\theta} \sup _{v \geqslant s>0} s^{1 / p_{0}} \bar{f}(s, M)\right)^{q} \frac{d t}{t}\right)^{\frac{1}{q}} \\
\quad=\left(\left(1 / p_{0}-1 / p_{1}\right) \int_{0}^{\infty}\left(t^{-\theta\left(\frac{1}{p_{0}}-\frac{1}{p_{1}}\right)} \sup _{t \geqslant s>0} s^{1 / p_{0}} \bar{f}(s, M)\right)^{q} \frac{d t}{t}\right)^{\frac{1}{q}} \\
\quad \geqslant\left(\left(1 / p_{0}-1 / p_{1}\right) \int_{0}^{\infty}\left(t^{\frac{1-\theta}{p_{0}}+\frac{\theta}{p_{1}}} \bar{f}(t, M)\right)^{q} \frac{d t}{t}\right)^{\frac{1}{q}}=c\|f\|_{N_{p q}(M, \mu)},
\end{gathered}
$$

which finishes the proof.

As a consequence we write the following interpolation result.

Corollary 2.1.1. Let $0<p_{0}<p_{1} \leqslant \infty, 0<q \leqslant \infty$, and $0<\theta<1$. Let $\left(X_{0}, X_{1}\right)$ be a compatible couple and $M$ be an arbitrary net in $\mathfrak{F}$. If a semiadditive operator $T$ acts as follows:

$$
\begin{aligned}
& T: X_{0} \rightarrow N_{p_{0} \infty}(M, \mu) \quad \text { with the norm } D_{0}, \\
& T: X_{1} \rightarrow N_{p_{1} \infty}(M, \mu) \quad \text { with the norm } D_{1},
\end{aligned}
$$

then we also have

$$
T:\left(X_{0}, X_{1}\right)_{\theta q} \rightarrow N_{p q}(M, \mu) \text { with the norm }\|T\| \leqslant c D_{0}^{1-\theta} D_{1}^{\theta},
$$

where $1 / p=(1-\theta) / p_{0}+\theta / p_{1}$. 
3. The STRONG-TYPE AND WEAK-TYPE BOUNDEDNESS OF INTEGRAL OPERATORS

Corollary 2.1.1 allows us to obtain the strong-type estimates using weaktype estimates. Next result gives the criterion for the integral operator to be a weak-type or quasi-weak type. First, we recall these definitions.

- An operator $T$ is a $(p, q)$ strong-type operator if $T: L_{p}(D, \nu) \rightarrow$ $L_{q}(\Omega, \mu)$.

- An operator $T$ is a $(p, q)$ weak-type operator if $T: L_{p}(D, \nu) \rightarrow$ $L_{q \infty}(\Omega, \mu)$.

- An operator $T$ is a $(p, q)$ quasi-weak-type operator if $T: L_{p 1}(D, \nu) \rightarrow$ $L_{q \infty}(\Omega, \mu)$.

Clearly, if $T$ is a $(p, q)$ strong-type operator, then it is a $(p, q)$ weak-type operator, and if $T$ is a $(p, q)$ weak-type operator, then it is a $(p, q)$ quasiweak-type operator (for $1 \leqslant p$ ).

Theorem 3.1. Let $1<q \leqslant \infty$ and let $M$ be any net from $\mathfrak{F}$. Suppose that $X(D, \nu)$ is a Banach space of measurable functions on $D$ and the integral operator

$$
T f(y)=\int_{D} K(x, y) f(x) d \nu_{x}
$$

acts from $X(D, \nu)$ into $N_{q \infty}(M, \mu)$.

Then the necessary and sufficient condition for $T$ to be bounded from $X(D, \nu)$ into $N_{q \infty}(M, \mu)$ is

$$
\sup _{e \in M}\left\|\frac{1}{(\mu(e))^{1 / q^{\prime}}} \int_{e} K(\cdot, y) d \mu\right\|_{X^{*}}<\infty,
$$

where $X^{*}$ is the associate space of $X$, i.e.,

$$
X^{*}=\left\{g:\|g\|_{X^{*}}=\sup _{\|f\|_{X} \leqslant 1}\left|\int_{D} g(x) f(x) d \nu\right|<\infty\right\} .
$$

Moreover,

$$
\|T\|_{X \rightarrow N_{q \infty}}=\sup _{e \in M}\left\|\frac{1}{\mu(e)^{1 / q^{\prime}}} \int_{e} K(\cdot, y) d \mu\right\|_{X^{*}} .
$$


Proof. Indeed, by the definition of the spaces $N_{q \infty}(M, \mu)$ and $X^{*}$, we have

$$
\begin{aligned}
\|T\|_{X \rightarrow N_{q \infty}(M, \mu)} & =\sup _{\|f\|_{X} \leqslant 1}\|T f\|_{N_{q \infty}(M, \mu)} \\
& =\sup _{\|f\|_{X} \leqslant 1} \sup _{e \in M} \frac{1}{(\mu(e))^{1 / q^{\prime}}}\left|\int_{e} \int_{D} K(x, y) f(x) d \nu d \mu\right| \\
& =\sup _{e \in M} \sup _{\|f\|_{X} \leqslant 1}\left|\int_{D} f(x) \frac{1}{(\mu(e))^{1 / q^{\prime}}} \int_{e} K(x, y) d \mu d \nu\right| \\
& =\sup _{e \in M}\left\|\frac{1}{\mu(e)^{1 / q^{\prime}}} \int_{e} K(\cdot, y) d \mu\right\|_{X^{*}}
\end{aligned}
$$

and the result follows.

Corollary 3.1.1. Let $1<p, q \leqslant \infty, M_{1}=\{e \subset \Omega: 0<\mu(e)<\infty\}$, and $M_{2}=\{w \subset D: 0<\nu(w)<\infty\}$. Then the necessary and sufficient condition for operator (3.1) to be $(p, q)$ quasi-weak-type operator is

$$
\sup _{e \in M_{1}, \omega \in M_{2}} \frac{1}{(\mu(e))^{1 / q^{\prime}}(\nu(w))^{1 / p}}\left|\int_{e} \int_{\omega} K(x, y) d \mu d \nu\right|<\infty .
$$

Moreover, for $0<r \leqslant 1$ we have

$$
\begin{aligned}
\|T\|_{L_{p r}(D, \nu) \rightarrow L_{q \infty}(\Omega, \mu)} & \asymp\|T\|_{L_{p 1}(D, \nu) \rightarrow L_{q \infty}(\Omega, \mu)} \\
& \asymp \sup _{e \in M_{1}, \omega \in M_{2}} \frac{1}{(\mu(e))^{1 / q^{\prime}}(\nu(w))^{1 / p}}\left|\int_{e} \int_{\omega} K(x, y) d \mu d \nu\right| .
\end{aligned}
$$

Proof. We use the properties of the net spaces and Theorem 3.1:

$$
\begin{aligned}
\|T\|_{L_{p r}(D, \nu) \rightarrow L_{q \infty}(\Omega, \mu)} & \leqslant C\|T\|_{L_{p 1}(D, \nu) \rightarrow L_{q \infty}(\Omega, \mu)} \\
& \asymp\|T\|_{L_{p 1}(D, \nu) \rightarrow N_{q \infty}\left(M_{1}, \mu\right)} \\
& =\sup _{e \in M_{1}} \frac{1}{(\mu(e))^{1 / q^{\prime}}}\left\|\int_{e} K(\cdot, y) d \mu\right\|_{L_{p^{\prime}, \infty}} \\
& \asymp \sup _{e \in M_{1}} \frac{1}{(\mu(e))^{1 / q^{\prime}}}\left\|\int_{e} K(\cdot, y) d \mu\right\|_{N_{p^{\prime}, \infty}\left(M_{2}, \nu\right)} \\
& =\sup _{e \in M_{1}} \sup _{\omega \in M_{2}} \frac{1}{(\mu(e))^{1 / q^{\prime}}} \frac{1}{(\nu(\omega))^{1 / p}}\left|\int_{e} \int_{\omega} K(x, y) d \mu d \nu\right| .
\end{aligned}
$$

To prove the inverse estimate of $\|T\|_{L_{p r} \rightarrow L_{q \infty}}$, we take the test function

$$
f_{0}(x)=\left(\frac{r}{p}\right)^{1 / r} \frac{\chi_{w}(x)}{(\nu(w))^{1 / p}}
$$

and note that

$$
\left\|f_{0}\right\|_{L_{p r}(D, \nu)}=1
$$

This completes the proof. 
Corollary 3.1.2. Let $1 \leqslant p \leqslant \infty, 1<q \leqslant \infty$, and $M_{1}=\{e \subset \Omega: 0<$ $\mu(e)<\infty\}$. Then the necessary and sufficient condition for the operator $T$ to be $(p, q)$ weak-type operator is

$$
\sup _{e \in M_{1}} \frac{1}{(\mu(e))^{1 / q^{\prime}}}\left(\int_{D}\left|\int_{e} K(x, y) d \mu\right|^{p^{\prime}} d \nu\right)^{\frac{1}{p^{\prime}}}<\infty .
$$

Proof. Similar to the proof of Corollary 3.1.1, we have

$$
\begin{aligned}
\|T\|_{L_{p}(D, \nu) \rightarrow L_{q \infty}(\Omega, \mu)} & \asymp\|T\|_{L_{p}(D, \nu) \rightarrow N_{q \infty}\left(M_{1}, \mu\right)} \\
& =\sup _{e \in M_{1}} \frac{1}{(\mu(e))^{1 / q^{\prime}}} \sup _{\|f\|_{p}=1}\left|\int_{D} f(x) \int_{e} K(x, y) d \mu d \nu\right| \\
& =\sup _{e \in M_{1}} \frac{1}{(\mu(e))^{1 / q^{\prime}}}\left(\int_{D}\left|\int_{e} K(x, y) d \mu\right|^{p^{\prime}} d \nu\right)^{\frac{1}{p^{\prime}}} .
\end{aligned}
$$

This completes the proof.

Remarks. We note that in the case of $\Omega=\mathbb{R}^{n}$ and when $\mu=\nu$ are $n$ dimensional Lebesgue measures, Corollaries 3.1.1 and 3.1.2 were obtained in $[\mathrm{KN}]$.

We also mention the following result from [CRS, 1.2]. Let $L \subset M(X)$ be a regular class, $T: L \rightarrow M(Y)$ be an order continuous sublinear operator (see definitions in [CRS]), and $0<q_{0} \leqslant 1,0<p_{0}<\infty$. Then

a) if $q_{0} \leqslant q_{1} \leqslant p_{1}<\infty$,

$$
\|T\|_{L_{p_{0} q_{0}} \rightarrow L_{p_{1} q_{1}}}=\sup _{\chi_{B}} \frac{\left\|T \chi_{B}\right\|_{L_{p_{1} q_{1}}}}{\left\|\chi_{B}\right\|_{L_{p_{0} q_{0}}}}
$$

b) if $q_{0}<p_{1}<q_{1} \leqslant \infty$,

$$
\|T\|_{L_{p_{0} q_{0}} \rightarrow L_{p_{1} q_{1}}} \leqslant\left(\frac{p_{1}}{p_{1}-q_{0}}\right)^{1 / q_{0}} \sup _{\chi_{B}} \frac{\left\|T \chi_{B}\right\|_{L_{p_{1} q_{1}}}}{\left\|\chi_{B}\right\|_{L_{p_{0} q_{0}}}} .
$$

By Bukhvalov's theorem (see $[\mathrm{Bu}]$ ), the order continuous linear operator is an integral. Generally speaking, the reverse is not true. So in the case of $q_{1}=\infty$, the results of Corollaries 3.1.1 and 3.1.2 supplement with estimates (1.2) and (3.3)-(3.4).

Corollary 3.1.3. Let $1<p \leqslant q<\infty$. Assume that $A \subset(0,1)^{2}$ is a convex set which is bounded by a closed polyline $\partial A$ with vertices $a_{1}, a_{2}, \ldots, a_{n}$. Let also $A_{0}:=\bar{A} \backslash\left\{a_{1}, \ldots, a_{n}\right\}$. Then the necessary and sufficient condition for an integral operator $T$ to be bounded from $L_{p}(D, \nu)$ into $L_{q}(\Omega, \mu)$ 
for any $\left(\frac{1}{p}, \frac{1}{q}\right) \in A_{0}$ is

$$
\sup _{e \in M_{1}, \omega \in M_{2}} \frac{1}{(\mu(e))^{1 / q^{\prime}}(\nu(w))^{1 / p}}\left|\int_{e} \int_{\omega} K(x, y) d \nu d \mu\right|<\infty
$$

for any $\left(\frac{1}{p}, \frac{1}{q}\right) \in \partial A \backslash\left\{a_{1}, \ldots, a_{n}\right\}$.

Proof. Note that for any point $b$ of the set $A_{0}$ we can find points $b_{0}, b_{1} \in$ $\partial A \backslash\left\{a_{1}, \ldots, a_{n}\right\}$ such that $b=(1-\theta) b_{0}+\theta b_{1}$ for $\theta \in(0,1)$. Then by Corollaries 3.1.1 and 3.1.2, condition (3.5) implies strong-type boundedness of the operator $A$ at the point $b$. The necessity follows from the fact that the strong-type boundedness implies the weak-type boundedness. The proof is now complete.

In a similar way one proves the following proposition.

Corollary 3.1.4. Let $1<p \leqslant q<\infty$. Let $A$ be an open set from $(0,1)^{2}$. Then the necessary and sufficient condition for an integral operator $T$ to be bounded from $L_{p}(D, \nu)$ to $L_{q}(\Omega, \mu)$ for any $\left(\frac{1}{p}, \frac{1}{q}\right) \in A_{0}$ is the accuracy of condition (3.5) for any $\left(\frac{1}{p}, \frac{1}{q}\right) \in A$.

Now we present a simple proof of the Hausdorff-Young-type inequality in the Lorentz spaces (see, for example, [SW, Ch. V]).

Example 3.1. Let $1<p<2$ and $0<q \leqslant \infty$. Then for the Fourier transform

we have

$$
\hat{f}(y)=\frac{1}{\sqrt{2 \pi}} \int_{\mathbb{R}} f(x) e^{-i y x} d x
$$

$$
C_{1}(p, q)\|H f\|_{L_{p q}(\mathbb{R}, d x)} \leqslant\|\hat{f}\|_{L_{p^{\prime} q}(\mathbb{R}, d x)} \leqslant C_{2}(p, q)\|f\|_{L_{p q}(\mathbb{R}, d x)}
$$

where $H f(x)$ is the following Hardy-type operator

$$
H f(x)=\frac{1}{|x|} \int_{-|x|}^{|x|} f(t) d t
$$

Proof. Let $1<p<\infty, M_{0}=\{[a, b]: a<b\}$, and $M_{1}=\{e \subset \mathbb{R}: 0<$ $m(e)=|e|<\infty\}$, where $m$ is the linear Lebesgue measure. Then

$$
\begin{aligned}
\sup _{\substack{w \in M_{0} \\
e \in M_{1}}} \frac{1}{|w|^{\frac{1}{p}}|e|^{\frac{1}{p}}}\left|\int_{w} \int_{e} e^{-i y x} d x d y\right| & \leqslant \sup _{\substack{w \in M_{0} \\
e \in M_{1}}} \frac{1}{|w|^{\frac{1}{p}}|e|^{\frac{1}{p}}} \int_{e} \frac{|\sin | w|x|}{|x|} d x \\
& \leqslant \sup _{\substack{w \in M_{0} \\
e \in M_{1}}} \frac{1}{|w|^{\frac{1}{p}}|e|^{\frac{1}{p}}} \int_{e} \min (|w|, 1 /|x|) d x \\
& \leqslant \sup _{e \in M_{1}} \frac{1}{|e|^{\frac{1}{p}}} \int_{e} \frac{1}{|x|^{\frac{1}{p^{\prime}}}} d x=p .
\end{aligned}
$$


Therefore, by Theorem 3.1, this implies for any $p \in(1, \infty)$

$$
\|\hat{f}\|_{N_{p^{\prime} \infty}\left(M_{0}, d x\right)} \leqslant C(p)\|f\|_{L_{p 1}(\mathbb{R}, d x)} .
$$

Let $1<p<\infty$. The last inequality implies for $1<p_{0}<p<p_{1}<\infty$ and $T f=\hat{f}$ that,

$$
\begin{array}{lll}
T: L_{p_{0} 1}(\mathbb{R}, d x) \rightarrow N_{p_{0}^{\prime} \infty}(M, \mu) & \text { with the norm } & C\left(p_{0}\right), \\
T: L_{p_{1} 1}(\mathbb{R}, d x) \rightarrow N_{p_{1}^{\prime} \infty}(M, \mu) & \text { with the norm } & C\left(p_{1}\right) .
\end{array}
$$

By Corollary 2.1.1,

$$
\|\hat{f}\|_{N_{p^{\prime} q}\left(M_{0}, d x\right)} \leqslant C(p, q)\|f\|_{L_{p q}(\mathbb{R}, d x)}
$$

and hence

$$
\|f\|_{N_{p^{\prime} q}\left(M_{0}, d x\right)} \leqslant C(p, q)\|\hat{f}\|_{L_{p q}(\mathbb{R}, d x)}
$$

and the left-hand side inequality in (3.6) follows.

To prove the right-hand side inequality, assume that $1<p<2$ and so $2<p^{\prime}<\infty$. Then, because of the fact that $\left|\hat{\chi}_{\omega}(y)\right| \leqslant|\omega|$ and by Plancherel's theorem, we get

$$
\begin{aligned}
\sup _{w \in M_{1}} \frac{1}{|w|^{\frac{1}{p}}}\left\|\int_{w} e^{-i y x} d x\right\|_{L_{p^{\prime}}} & =\sup _{w \in M_{1}} \frac{1}{|w|^{\frac{1}{p}}}\left(\int_{\mathbb{R}}\left|\hat{\chi}_{w}\right|^{2}\left|\hat{\chi}_{w}\right|^{p^{\prime}-2} d x\right)^{\frac{1}{p^{\prime}}} \\
& \leqslant \sup _{w \in M_{1}} \frac{|w|^{1-2 / p^{\prime}}}{|w|^{\frac{1}{p}}}\left(\int_{\mathbb{R}}\left|\hat{\chi}_{w}\right|^{2} d x\right)^{\frac{1}{p^{\prime}}} \\
& =\sup _{w \in M_{1}} \frac{|w|^{\frac{1}{p^{\prime}}}|w|^{1-\frac{2}{p^{\prime}}}}{|w|^{\frac{1}{p}}}=1 .
\end{aligned}
$$

Hence for any $p \in(1,2)$ we write

$$
\|\hat{f}\|_{L_{p^{\prime} \infty}(\mathbb{R}, d x)} \asymp\|\hat{f}\|_{N_{p^{\prime} \infty}\left(M_{1}, d x\right)} \leqslant\|f\|_{L_{p}(\mathbb{R}, d x)} .
$$

Thus

$$
\|\hat{f}\|_{L_{p^{\prime} q}(\mathbb{R}, d x)} \asymp\|\hat{f}\|_{N_{p^{\prime} q}\left(M_{1}, d x\right)} \leqslant C\|f\|_{L_{p q}(\mathbb{R}, d x)} .
$$

The proof is now complete.

Example 3.2. Let $1<p<2$ and $0<q \leqslant \infty$. Then if $|f(x)| \leqslant C|H f(x)|$, then we have

$$
\|\hat{f}\|_{L_{p^{\prime} q}(\mathbb{R}, d x)} \asymp\|f\|_{L_{p q}(\mathbb{R}, d x)} .
$$

Let $2<p<\infty$ and $0<q \leqslant \infty$. Then if $|\hat{f}(x)| \leqslant C|H \hat{f}(x)|$, then we also have (3.7). 
If we assume a stronger condition like monotonicity or quasi-monotonicity $^{1}$ of $f(x)$ and $f(-x)$ ( or of $\hat{f}(x)$ and $\hat{f}(-x)$ ) on $\mathbb{R}_{+}$, then (3.7) is true for $p \in(1, \infty)$. The proof easily follows from the proof in Example 3.1, and duality arguments (see also Sagher's papers [Sa] and [BS]).

Now we present the main result in this section which is the sufficient conditions for an integral operator to be bounded in the Lorentz spaces.

Let now $e \in M_{1}$ and $w \in M_{2}$. Define the following function

$$
F(e, \omega):=F(e, \omega ; K):=\frac{1}{\nu(\omega)^{\frac{1}{p}}} \frac{1}{\mu(e)^{\frac{1}{q^{\prime}}}}\left|\int_{e} \int_{\omega} K(x, y) d \nu d \mu\right| .
$$

Theorem 3.2. Let $1 \leqslant r, \theta, h \leqslant \infty$, and $1 / r+1=1 / h+1 / \theta$. Let also $M_{1}=\{e \subset \Omega: 0<\mu(e)<\infty\}, M_{2}=\{w \subset D: 0<\nu(w)<\infty\}$. If there exists $\gamma>0$ such that

$$
B=\left(\int_{0}^{\infty}\left(\sup _{\mu(e) / \nu \gamma(w)=t} F(e, w)\right)^{h} \frac{d t}{t}\right)^{\frac{1}{h}}<\infty \quad \text { if } \quad h<\infty
$$

and

$$
B=\sup _{e \in M_{1}, \omega \in M_{2}} F(e, w)<\infty \quad \text { if } h=\infty,
$$

then the integral operator

$$
T f(y)=\int_{D} K(x, y) f(x) d \nu_{x}
$$

is bounded from $L_{p \theta}(D, \nu)$ into $L_{q r}(\Omega, \mu), 1<p, q<\infty$, and

$$
\|T\|_{L_{p \theta} \rightarrow L_{q r}} \leqslant C(p, q) B .
$$

Moreover, in the case of $r=\infty$ and $\theta=1$, the condition $B<\infty$ is also necessary.

Proof. We define

$$
\Phi(s, t):=\sup _{\substack{\mu(e)=t \\ e \in M_{1}}} \sup _{\substack{\nu(w)=s \\ w \in M_{2}}} F(e, w) .
$$

First, let us show the accuracy of

$$
\sup _{\substack{\mu(e)=t \\ e \in M_{1}}} \frac{1}{\mu(e)}\left|\int_{e} T f(y) d \mu\right| \leqslant C t^{-1 / q} \int_{0}^{\infty} s^{1 / p-1} f^{*}(s) \Phi(s, t) d s .
$$

\footnotetext{
${ }^{1}$ A function $f(x)$ on $\mathbb{R}_{+}$is quasi-monotone if there exists $\tau>0$ such that $f(x) / x^{\tau}$ is monotone decreasing.
} 
Indeed, using the Hardy inequality $\left(\int f g \leqslant \int f^{*} g^{*}\right)$ and the monotonicity of $K^{*}$, we get

$$
\begin{gathered}
\sup _{\substack{\mu(e)=t \\
e \in M_{1}}} \frac{1}{\mu(e)}\left|\int_{e} T f(y) d \mu\right|=t^{-1 / q} \sup _{\substack{\mu(e)=t \\
e \in M_{1}}} \frac{1}{(\mu(e))^{1 / q^{\prime}}}\left|\int_{D} f(x) \int_{e} K(x, y) d \mu d \nu\right| \\
\leqslant t^{-1 / q} \sup _{\substack{\mu(e)=t \\
e \in M_{1}}} \frac{1}{(\mu(e))^{1 / q^{\prime}}} \int_{0}^{\infty} f^{*}(s)\left(\int_{e} K(\cdot, y) d \mu\right)^{*}(s) d s \\
\leqslant t^{-1 / q} \sup _{\substack{\mu(e)=t \\
e \in M_{1}}} \frac{1}{(\mu(e))^{1 / q^{\prime}}} \int_{0}^{\infty} f^{*}(s)\left(\int_{e} K(\cdot, y) d \mu\right)^{* *}(s) d s .
\end{gathered}
$$

Further, by Lemma 2.1, for $g(x) \equiv \int_{e} K(x, y) d \mu$ we write

$$
g^{* *}(s) \leqslant 4 \sup _{\substack{\nu(w)=s / 3 \\ w \in M_{2}}} \frac{1}{\nu(w)}\left|\int_{w} g(x) d \nu\right| .
$$

Then the left hand side of (3.10) is bounded by

$$
\begin{array}{r}
12 t^{-1 / q} \int_{0}^{\infty} f^{*}(3 s) \sup _{\substack{\mu(e)=t \\
e \in M_{1}}} \sup _{\substack{\nu(w)=s \\
w \in M_{2}}} \frac{1}{(\mu(e))^{1 / q^{\prime}}} \frac{1}{(\nu(w))}\left|\int_{w} \int_{e} K(x, y) d \mu d \nu\right| d s \\
\leqslant 12 t^{-1 / q} \int_{0}^{\infty} s^{1 / p-1} f^{*}(s) \Phi(s, t) d s
\end{array}
$$

and (3.10) follows.

Then, using Lemma 2.1 and (3.10), we have

$$
\begin{aligned}
\|T f\|_{L_{q r}} & \asymp\left(\int_{0}^{\infty}\left(t^{1 / q}(T f)^{* *}(t)\right)^{r} d t / t\right)^{1 / r} \\
& \leqslant C\left(\int_{0}^{\infty}\left(t^{1 / q} \sup _{\substack{\mu(e)=t \\
e \in M_{1}}} \frac{1}{\mu(e)}\left|\int_{e} T f(y) d \mu\right|\right)^{r} \frac{d t}{t}\right)^{1 / r} \\
& \leqslant C\left(\int_{0}^{\infty}\left(\int_{0}^{\infty} s^{1 / p-1} f^{*}(s) \Phi(s, t) d s\right)^{r} \frac{d t}{t}\right)^{1 / r} .
\end{aligned}
$$

Further we use the following representation

$$
s^{1 / p} f^{*}(s) \Phi(s, t)=\left(\left(s^{1 / p} f^{*}\right)^{1-\theta / r}\right)\left(\left(s^{1 / p} f^{*}\right)^{\theta / r} \Phi(s, t)\right) .
$$


Then by the Hölder inequality with exponents $h$ and $h^{\prime}=\left(\frac{1}{\theta}-\frac{1}{r}\right)^{-1}$, we write $^{2}$

$$
\begin{aligned}
\|T f\|_{L_{q r}} \leqslant & \left(\int _ { 0 } ^ { \infty } \left[\left(\int_{0}^{\infty}\left(s^{1 / p} f^{*}(s)\right)^{\theta} \frac{d s}{s}\right)^{1 / \theta-1 / r}\right.\right. \\
& \left.\left.\left(\int_{0}^{\infty}\left(s^{1 / p} f^{*}(s)\right)^{\theta h / r} \Phi^{h}(s, t) \frac{d s}{s}\right)^{1 / h}\right]^{r} \frac{d t}{t}\right)^{1 / r} \\
= & \|f\|_{L_{p \theta}}^{1-\theta / r}\left(\int_{0}^{\infty}\left(\int_{0}^{\infty}\left[s^{1 / p} f^{*}(s)\right]^{\theta h / r} \Phi^{h}(s, t) \frac{d s}{s}\right)^{r / h} \frac{d t}{t}\right)^{1 / r} .
\end{aligned}
$$

Changing the variables $s \rightarrow s t^{\gamma}$, using the Minkowski inequality and then changing the variables $s t^{\gamma} \rightarrow t$ again, we get

$$
\begin{aligned}
\|T f\|_{L_{q r}} & \leqslant C\|f\|_{L_{p \theta}}^{1-\theta / r}\left(\int_{0}^{\infty}\left(\int_{0}^{\infty}\left[\left(s t^{\gamma}\right)^{1 / p} f^{*}\left(s t^{\gamma}\right)\right]^{\theta h / r} \Phi^{h}\left(s t^{\gamma}, t\right) \frac{d s}{s}\right)^{r / h} \frac{d t}{t}\right)^{1 / r} \\
& \leqslant C\|f\|_{L_{p \theta}}^{1-\theta / r}\left(\int_{0}^{\infty}\left(\int_{0}^{\infty}\left[\left(s t^{\gamma}\right)^{1 / p} f^{*}\left(s t^{\gamma}\right)\right]^{\theta} \Phi^{r}\left(s t^{\gamma}, t\right) \frac{d t}{t}\right)^{h / r} \frac{d s}{s}\right)^{1 / h} \\
& =C\|f\|_{L_{p \theta}}^{1-\theta / r}\left(\int_{0}^{\infty}\left(\int_{0}^{\infty}\left[t^{1 / p} f^{*}(t)\right]^{\theta} \Phi^{r}\left(t,(t / s)^{\frac{1}{\gamma}}\right) \frac{d t}{t}\right)^{h / r} \frac{d s}{s}\right)^{1 / h} .
\end{aligned}
$$

Noting

$$
\Phi\left(t,(t / s)^{\frac{1}{\gamma}}\right) \leqslant \sup _{\sigma / \tau^{\gamma}=s} \Phi(\sigma, \tau)
$$

we write

$$
\begin{aligned}
\|T f\|_{L_{q r}} & \leqslant C\|f\|_{L_{p \theta}}\left(\int_{0}^{\infty}\left(\sup _{\sigma / \tau^{\gamma}=s} \Phi(\sigma, \tau)\right)^{h} \frac{d s}{s}\right)^{1 / h} \\
& =C\|f\|_{L_{p \theta}}\left(\int_{0}^{\infty}\left(\sup _{\mu(e) / \nu^{\gamma}(w)=s} F(e, w)\right)^{h} \frac{d s}{s}\right)^{1 / h} .
\end{aligned}
$$

If $\theta=1$ and $r=\infty$, the necessity of condition (3.9) follows from Corollary 3.1.1. The proof is now complete.

\footnotetext{
${ }^{2}$ In the case of $h=1$ or $h=\infty$ the proof is similar. We use the usual adjustment of $\left(\int|g|^{q}\right)^{1 / q}$ when $q=\infty$.
} 
Remark 3.1. From the proof of Theorem 3.2 one can observe that condition (3.8) can be changed to the following condition: there exist $c>0$ and $\gamma>0$ such that

$$
B=\left(\int_{0}^{\infty}\left(\sup _{\mu(e) / c \nu^{\gamma}(w)=t} F(e, w)\right)^{h} \frac{d t}{t}\right)^{\frac{1}{h}}<\infty
$$

\section{Convolution operator in Lebesgue spaces}

First, we study the $(p, q)$-strong-type convolution operators. We will need the following lemma.

Lemma 4.1. Let $f, g$, and $K$ be measurable functions on $\mathbb{R}^{n}$. Then

$$
\int_{\mathbb{R}} f(y) \int_{\mathbb{R}} g(x) K(x-y) d x d y \leqslant \int_{0}^{\infty} f^{*}(s) \int_{0}^{\infty} g^{*}(t) K^{* *}(|s-t|) d t d s,
$$

where $K^{* *}(|s-t|)=\frac{1}{|s-t|} \int_{0}^{|s-t|} K^{*}(\xi) d \xi$.

Proof. We use the Hardy inequality on rearrangements:

$$
\begin{aligned}
\left|\int_{-\infty}^{\infty} g(y)(f * K)(y) d y\right| & \leqslant \int_{0}^{\infty} g^{*}(s)(f * K)^{* *}(s) d s \\
& =\int_{0}^{\infty} g^{*}(s) \sup _{|e|=s} \frac{1}{|e|} \int_{e}\left|\int_{-\infty}^{\infty} K(y-x) f(x) d x\right| d y d s \\
& \leqslant \int_{0}^{\infty} g^{*}(s) \sup _{|e|=s} \int_{0}^{\infty} f^{*}(t) \sup _{|w|=t} \frac{1}{|e|} \frac{1}{|w|} \\
& \leqslant \int_{w}^{\infty} \int_{e}|K(y-x)| d y d x d t d s \\
& \int_{w} \int_{e}|K(y-x)| d y d x d t d s .
\end{aligned}
$$

Then the inequality

$$
\sup _{\substack{|w|=t \\|e|=s}} \frac{1}{|e|} \frac{1}{|w|} \int_{w} \int_{e}|K(x-y)| d x d y \leqslant K^{* *}(\max (s, t)) \leqslant K^{* *}(|s-t|)
$$

finishes the proof of Lemma 4.1. 
Theorem 4.1. Let $\alpha, \beta \in[0,1), 1<p, q<\infty$, and $0<\frac{1}{r}=1-\frac{1-\alpha}{p^{\prime}}-\frac{1-\beta}{q}$. Suppose that measures $\mu$ and $\nu$ are defined as follows

$$
\mu(e)=\int_{e} \frac{d y}{|y|^{\beta}}, \quad \nu(\omega)=\int_{\omega} \frac{d x}{|x|^{\alpha}}
$$

Then the norm of the convolution operator

$$
A f(y)=\int_{-\infty}^{+\infty} K(y-x) f(x) d \nu
$$

satisfies

$$
\|A\|_{L_{p}(\mathbb{R}, \nu) \rightarrow L_{q}(\mathbb{R}, \mu)} \leqslant c \sup _{e \in M^{*}} \frac{1}{|e|^{\frac{1}{r}}}\left|\int_{e} K(t) d t\right|=: c B,
$$

where $|e|$ is the linear measure of e. Moreover, if the kernel $K(t)$ is nonnegative, then

$$
c \sup _{d>0} \frac{1}{d^{\frac{1}{r}}} \int_{-d}^{d} K(t) d t \leqslant\|A\|_{L_{p}(\mathbb{R}, \nu) \rightarrow L_{q}(\mathbb{R}, \mu)} .
$$

Proof. Denote

$$
\gamma=\frac{1-\beta}{1-\alpha} \quad \text { and } \quad \delta=\frac{(1-\alpha)^{\gamma}}{1-\beta}
$$

By Lemma 4.1,

$$
\begin{aligned}
& \sup _{\mu(e)=\delta(\nu(\omega))^{\gamma} t} F(e, \omega)=\sup _{\mu(e)=\delta(\nu(\omega))^{\gamma} t} \frac{1}{\mu(e)^{\frac{1}{q^{\prime}}} \nu(w)^{\frac{1}{p}}} \int_{e} \int_{\omega} \frac{K(x-y) d x d y}{|x|^{\alpha}|y|^{\beta}} \\
& \leqslant \sup _{\mu(e)=\delta(\nu(\omega))^{\gamma} t} \frac{1}{\mu(e)^{\frac{1}{q^{\prime}}} \nu(w)^{\frac{1}{p}}} \int_{0}^{|e|} \int_{0}^{|\omega|} \frac{1}{|x|^{\alpha}|y|^{\beta}} \\
& \left(\frac{1}{|x-y|} \int_{0}^{|x-y|} K^{*}(t) d t\right) d x d y \\
& \leqslant B \sup _{\mu(e)=\delta(\nu(\omega))^{\gamma} t} \frac{1}{\mu(e)^{\frac{1}{q^{\prime}}} \nu(w)^{\frac{1}{p}}} \\
& \int_{0}^{|e|} \int_{0}^{|\omega|} \frac{d x d y}{|x-y|^{1-\frac{1}{r}}|x|^{\alpha}|y|^{\beta}} \\
& =B \sup _{a>0} \frac{1}{\left(a t^{\frac{1}{1-\beta}}\right)^{\frac{1-\beta}{q^{\prime}}}\left(a^{(1-\alpha)}\right)^{\frac{1}{p}}} \\
& \int_{0}^{a t^{\frac{1}{1-\beta}}} \int_{0}^{a} \frac{d x d y}{|x-y|^{1-\frac{1}{r}}|x|^{\alpha}|y|^{\beta}} .
\end{aligned}
$$


In the last inequality we have used the fact that

$$
\mu\left(\left[0, a t^{\frac{1}{1-\beta}}\right]\right)=\delta(\nu([0, a]))^{\gamma} t .
$$

Furthermore, we remark that

$$
\begin{gathered}
\sup _{a>0} \frac{t^{-1 / q^{\prime}}}{a^{\frac{1-\alpha}{p}+\frac{1-\beta}{q^{\prime}}}} \int_{0}^{a t^{\frac{1}{1-\beta}}} \int_{0}^{a} \frac{d x d y}{|x-y|^{1-\frac{1}{r}}|x|^{\alpha}|y|^{\beta}}= \\
t^{\frac{1}{q}} \int_{0}^{1} \int_{0}^{1} \frac{d z d s}{\left|z-t^{\frac{1}{1-\beta}} s\right|^{\theta} z^{\alpha} s^{\beta}} .
\end{gathered}
$$

Therefore,

$$
\begin{aligned}
\left(\int_{0}^{\infty}\left(\sup _{\mu(e)=\delta(\nu(\omega))^{\gamma} t} F(e, \omega)\right)^{h} \frac{d t}{t}\right)^{\frac{1}{h}} & \\
\leqslant & B\left(\int_{0}^{\infty}\left(t^{\frac{1}{q}} \int_{0}^{1} \int_{0}^{1} \frac{d z d s}{\left|z-t^{\frac{1}{1-\beta}} s\right|^{1-\frac{1}{r}} z^{\alpha} s^{\beta}}\right)^{h} \frac{d t}{t}\right)^{\frac{1}{h}} \\
& =C B\left(\int_{0}^{\infty}\left(y^{\frac{1-\beta}{q}} \int_{0}^{1} \int_{0}^{1} \frac{d z d s}{|z-y s|^{1-\frac{1}{r}} z^{\alpha} s^{\beta}}\right)^{h} \frac{d y}{y}\right)^{\frac{1}{h}}
\end{aligned}
$$

and by the Minkowski inequality,

$$
\begin{aligned}
& \leqslant C B \int_{0}^{1} \int_{0}^{1}\left(\int_{0}^{\infty}\left(\frac{y^{\frac{1-\beta}{q}}}{|z-y s|^{1-\frac{1}{r}} z^{\alpha} s^{\beta}}\right)^{h} \frac{d y}{y}\right)^{\frac{1}{h}} d z d s \\
& =C B\left(\int_{0}^{1} \int_{0}^{1}\left(\int_{0}^{\infty}\left(\frac{x z}{s}\right)^{\frac{1-\beta}{q}} \frac{1}{z^{1-\frac{1}{r}}|1-x|^{1-\frac{1}{r}}}\right)^{h} \frac{d x}{x}\right)^{\frac{1}{h}} \frac{d z}{z^{\alpha}} \frac{d s}{s^{\beta}} \\
& =C B\left(\int_{0}^{1} z^{\frac{1-\alpha}{p}-1} d z\right)\left(\int_{0}^{1} s^{\frac{1-\beta}{q^{\prime}}-1} d s\right)\left(\int_{0}^{\infty}\left(\frac{x^{\frac{1-\beta}{q}}}{|1-x|^{\frac{1-\alpha}{p^{\prime}}+\frac{1-\beta}{q}}}\right)^{h} \frac{d x}{x}\right)^{\frac{1}{h}} \\
& =C_{1} B .
\end{aligned}
$$

Thus, remark 3.1 implies sufficient part.

Let us prove the estimate of $\|A\|$ from below. Suppose now that the operator $A$ is bounded from $L_{p}(\mathbb{R}, \nu)$ into $L_{q}(\mathbb{R}, \mu)$, i.e.,

$$
\|A\|_{L_{p}(\mathbb{R}, \nu) \rightarrow L_{q}(\mathbb{R}, \mu)}=\sup _{\substack{\|g\|_{L_{q^{\prime}}(\mathbb{R}, \mu)=1}=1 \\\|f\|_{L_{p}(\mathbb{R}, \nu)}=1}} \int_{\mathbb{R}} g(y) \int_{\mathbb{R}} K(y-x) f(x)|x|^{-\alpha}|y|^{-\beta} d x d y<\infty .
$$


For any $d>0$ we define the test functions:

$$
g_{0}(y)=\chi_{[-d, d]}(y) /\left\|\chi_{[-d, d]}\right\|_{L_{q^{\prime}}(\mathbb{R}, \mu)}=C_{2} d^{-\frac{1-\beta}{q^{\prime}}} \chi_{[-d, d]}(y)
$$

and

$$
\begin{aligned}
f_{0}(x) & =|x|^{\alpha} \chi_{[-2 d, 2 d]}(x) /\left\||x|^{\alpha} \chi_{[-2 d, 2 d]}\right\|_{L_{p}(\mathbb{R}, \nu)} \\
& =|x|^{\alpha} \chi_{[-2 d, 2 d]}(x)\left(\int_{-2 d}^{2 d}\left(|y|^{\alpha}\right)^{p}|y|^{-\alpha} d y\right)^{-\frac{1}{p}} \\
& =C_{3} d^{\frac{1-\alpha}{p^{\prime}}-1}|x|^{\alpha} \chi_{[-2 d, 2 d]}(x) .
\end{aligned}
$$

Therefore,

$$
\|A\|_{L_{p}(\mathbb{R}, \nu) \rightarrow L_{q}(\mathbb{R}, \mu)} \geqslant C_{4} \frac{1}{d^{\frac{1-\beta}{q^{\prime}}+1-\frac{1-\alpha}{p^{\prime}}}} \int_{-d}^{d}|y|^{-\beta} \int_{-2 d}^{2 d} K(y-x) d x d y
$$

Now using $K \geqslant 0$, we get for any $y \in[-d, d]:[-d, d] \subset[-2 d, 2 d]+y$ and

$$
\|A\|_{L_{p}(\mathbb{R}, \nu) \rightarrow L_{q}(\mathbb{R}, \mu)} \geqslant C_{5} \frac{1}{d^{1-\frac{1-\alpha}{p^{\prime}}-\frac{1-\beta}{q}}} \int_{-d}^{d} K(z) d z
$$

Taking into account arbitrary choice of $d>0$, we get (4.2). The proof is now complete.

Now we introduce the following concept.

Definition. A measurable function $f(x)$ on $\mathbb{R}$ is said to be weak monotone if for any $x \in \mathbb{R}$ one has

$$
|f(x)| \leqslant C \sup _{d \geqslant|x|} \frac{1}{d}\left|\int_{-d}^{d} f(y) d y\right| .
$$

Note that if one of nonnegative functions $f(x)$ or $f(-x)$ is monotone decreasing or, more general, quasi-monotone on $\mathbb{R}_{+}$, then $f(x)$ is a weak monotone. On the other hand, the following functions are not quasi-monotone but weak monotone:

$$
\frac{|\sin x|}{|x|^{\alpha}}, \quad \frac{\cos x}{|x|^{\alpha}}, \quad \alpha \in(0,1)
$$

Corollary 4.1.1. Let us assume that all conditions of Theorem 4.1 hold and $K$ is a nonnegative weak monotone function on $\mathbb{R}$. Then the necessary and sufficient condition for the convolution operator $A f$ to be bounded from $L_{p}(\mathbb{R}, \nu)$ into $L_{q}(\mathbb{R}, \mu)$ is

$$
\sup _{d>0} \frac{1}{d^{\frac{1}{r}}} \int_{-d}^{d} K(t) d t<\infty
$$


Proof. Since $K$ is a weak monotone, we obtain

$$
\begin{aligned}
\sup _{e \in M^{*}} \frac{1}{|e|^{\frac{1}{r}}}\left|\int_{e} K(t) d t\right| & \leqslant c \sup _{e \in M^{*}} \frac{1}{|e|^{\frac{1}{r}}}\left|\int_{e} \sup _{d \geqslant|t|} \frac{1}{d} \int_{-d}^{d} K(s) d s d t\right| \\
& \leqslant c \sup _{d>0} \frac{1}{d^{\frac{1}{r}}} \int_{-d}^{d} K(s) d s \sup _{e \in M^{*}} \frac{1}{|e|^{\frac{1}{r}}}\left|\int_{e} \frac{1}{|t|^{1-1 / r}} d t\right| \\
& \leqslant c \sup _{d>0} \frac{1}{d^{\frac{1}{r}}} \int_{-d}^{d} K(s) d s,
\end{aligned}
$$

and the required follows form Theorem 4.1.

From the proof of Theorem 4.1 one can obtain its analogue for functions on an interval.

Theorem 4.2. Let $\alpha, \beta \in[0,1), 1<p, q<\infty, 1+\frac{1}{q}=\frac{1}{p}+\frac{1}{h}$, and $0<\frac{1}{r}=1-\frac{1-\alpha}{p^{\prime}}-\frac{1-\beta}{q}$. Let $K$ be a nonnegative weak monotone function on $[-2 a, 2 a]$. Suppose that the measures $\mu$ and $\nu$ are defined as follows

and

$$
\mu(e)=\int_{e} \frac{d y}{|y|^{\beta}}, \quad \nu(\omega)=\int_{\omega} \frac{d x}{|x|^{\alpha}}
$$

Then

$$
A f(y)=\int_{-a}^{a} K(x-y) f(x) \frac{d x}{|x|^{\alpha}}
$$

$$
\|A\|_{L_{p}([-a, a], \nu) \rightarrow L_{q}([-a, a], \mu)} \asymp \sup _{2 a \geqslant d>0} \frac{1}{d^{\frac{1}{r}}} \int_{-d}^{d} K(t) d t<\infty .
$$

As applications of this result, we obtain the sharp increase rate for the norm of the Cesàro operator from $L_{p}\left(|x|^{\gamma}\right)$ into $L_{q}\left(|x|^{-\beta}\right)$ and a weighted version of the Nikol'skii inequality for trigonometrical polynomials (see also $[\mathrm{MT}])$.

Corollary 4.2.1. The Fejér kernel is defined as

$$
F_{n}(x)=\frac{1}{n} \sum_{k=0}^{n-1} D_{k}(x)=\frac{1}{n} \sum_{k=0}^{n-1} \sum_{\nu=-k}^{k} e^{2 \pi i \nu x}=\frac{1}{n} \frac{\sin ^{2} \pi(2 n-1) x}{\sin ^{2} \pi x} .
$$

It is well known that

$$
F_{n}(x) \geqslant 0 \quad \text { and } \quad \int_{-1 / 2}^{1 / 2} F_{n}(x) d x=1 .
$$


The Cesàro sum of 1-periodic function $f$ is written by

$$
\sigma_{n}(f, y)=\int_{-1 / 2}^{1 / 2} f(x) F_{n}(x-y) d x
$$

and the $n$-th partial sum of the Fourier series of $f$ is

$$
S_{n}(f, y)=\int_{-1 / 2}^{1 / 2} f(x) D_{n}(x-y) d x .
$$

Then for $1<p, q<\infty, 0 \leqslant \beta<1,0 \leqslant \gamma<p-1$, we have

$$
\begin{aligned}
& \sup _{\|f\|_{L_{p}([-1 / 2,1 / 2],|x| \gamma)=1}}\left\|\sigma_{n}(f)\right\|_{L_{q}\left([-1 / 2,1 / 2],|x|^{-\beta}\right)} \asymp n^{\frac{1+\gamma}{p}-\frac{1-\beta}{q}}, \\
& \sup _{\|f\|_{L_{p}([-1 / 2,1 / 2],|x| \gamma)=1}}\left\|S_{n}(f)\right\|_{L_{q}\left([-1 / 2,1 / 2],|x|^{-\beta}\right)} \leqslant C n^{\frac{1+\gamma}{p}-\frac{1-\beta}{q}} .
\end{aligned}
$$

where $\|f\|_{L_{q}\left([-1 / 2,1 / 2],|x|^{-\beta}\right)}=\left(\int_{-1 / 2}^{1 / 2}|f(x)|^{q}|x|^{-\beta} d x\right)^{\frac{1}{q}}$

Proof. One can note that

$$
F_{n}(x) \leqslant \frac{c}{|x|} \int_{-|x|}^{|x|} F_{n}(t) d t, \quad|x| \in(0,1 / 2] .
$$

Indeed,

$$
\frac{(\sin (2 n-1) \pi x)^{2}}{n(\sin \pi x)^{2}} \leqslant \frac{\min \left((2 n-1)^{2}, 1 / x^{2}\right)}{n} \leqslant 4 \min (n, 1 /|x|),
$$

If $x \geqslant 1 /(2 n-1)$,

$$
\begin{aligned}
\frac{1}{x} \int_{0}^{x} \frac{(\sin (2 n-1) \pi t)^{2}}{n(\sin \pi t)^{2}} d t & \geqslant \frac{c}{x n} \sum_{k=1}^{m_{0}}\left(\frac{(2 n-1)}{k}\right)^{2} \\
& =\frac{c}{x} \sum_{k=1}^{m_{0}} \frac{1}{k^{2}} \int_{k-1}^{k /(2 n-1)}(\sin \pi y)^{2} d y \\
& \left.\geqslant \frac{c}{x} \sum_{k=1}^{m_{0}} \frac{1}{k^{2}} \geqslant \frac{c}{x} \geqslant c \sin (2 n-1) \pi t\right)^{2} d t
\end{aligned}
$$

If $0<x<1 /(2 n-1)$,

$$
\frac{1}{x} \int_{0}^{x} \frac{(\sin (2 n-1) \pi t)^{2}}{n(\sin \pi t)^{2}} d t \geqslant \frac{n}{3} \geqslant \frac{\min (n, 1 / x)}{3} .
$$


Therefore, (4.5) follows. Then Theorem 4.2 implies

$$
\|A\|_{L_{p}([-a, a], \nu) \rightarrow L_{q}([-a, a], \mu)} \asymp \sup _{2 a \geqslant d>0} \frac{1}{d^{\frac{1}{r}}} \int_{-d}^{d} K(t) d t<\infty .
$$

So, for $\alpha=\gamma /(p-1)$ we get

$$
\begin{aligned}
\sup _{\|f\|_{L_{p}\left([-\pi, \pi],|x|^{\gamma}\right)=1}}\left\|\sigma_{n}(f)\right\|_{L_{q}\left([-\pi, \pi],|x|^{-\beta}\right)} & \\
& =\sup _{\|g\|_{L_{p}\left([-\pi, \pi],|x|^{-\alpha)}=1\right.}}\left\|\sigma_{n}\left(g(\cdot)|\cdot|^{-\alpha}\right)\right\|_{L_{q}\left([-\pi, \pi],|x|^{-\beta}\right)} \\
& \asymp \sup _{2 a \geqslant d>0} \frac{1}{d^{1-\frac{1-\alpha}{p^{\prime}}-\frac{1-\beta}{q}}} \int_{-d}^{d} F_{n}(t) d t .
\end{aligned}
$$

Let us now show that

$$
\sup _{2 a \geqslant d>0} \frac{1}{d^{\frac{1}{r}}} \int_{-d}^{d} F_{n}(t) d t \asymp n^{1 / r}
$$

From the proof above we have

$$
\sup _{2 a \geqslant d>0} \frac{1}{d^{1 / r}} \int_{-d}^{d} F_{n}(t) d t \geqslant c \sup _{2 a \geqslant d \geqslant 1 / n} d^{1-1 / r} \min (n, 1 / d)=c n^{1 / r}
$$

On the other hand,

$$
\sup _{2 a \geqslant d>0} \frac{1}{d^{1 / r}} \int_{-d}^{d} F_{n}(t) d t \leqslant c \sup _{2 a \geqslant d>0} \frac{1}{d^{1 / r}} \int_{0}^{d} \min (n, 1 / t) d t .
$$

Then we write $\sup _{1 / n \geqslant d>0} d^{-1 / r} d n=n^{1 / r}$ and

$$
\begin{aligned}
& \sup _{2 a \geqslant d>1 / n} \frac{1}{d^{1 / r}}\left(\int_{0}^{1 / n}+\int_{1 / n}^{d}\right) \min (n, 1 / t) d t \\
& \quad \leqslant c \sup _{2 a \geqslant d>1 / n} d^{-1 / r}+c n^{1 / r} \sup _{2 a \geqslant d>1 / n}(n d)^{-1 / r}(1+\ln (n d)) \leqslant c n^{1 / r} .
\end{aligned}
$$

Therefore, the following is true: if $1<p, q<\infty, 0 \leqslant \beta<1$, and $0 \leqslant \gamma<$ $p-1$, then

$$
\sup _{\|f\|_{L_{p}\left([-\pi, \pi],|x|^{\gamma}\right)=1}}\left\|\sigma_{n}(f)\right\|_{L_{q}\left([-\pi, \pi],|x|^{-\beta}\right)} \asymp n^{1-\frac{1-\alpha}{p^{\prime}}-\frac{1-\beta}{q}}=n^{\frac{1+\gamma}{p}-\frac{1-\beta}{q}} .
$$


Let us prove now the estimate for $S_{n}(f, x)$. We consider the following operator

$$
\begin{aligned}
\left|S_{n}\right|(f, y) & =\int_{-1 / 2}^{1 / 2} f(x)\left|D_{n}(x-y)\right| d x \\
& =\int_{-1 / 2}^{1 / 2} f(x)\left|\frac{\sin (2 n-1) \pi(x-y)}{\sin \pi(x-y)}\right| d x .
\end{aligned}
$$

It is clear that $\left\|S_{n}(f)\right\|_{L_{p} \rightarrow L_{q}} \leqslant\left\|\left|S_{n}\right|(f)\right\|_{L_{p} \rightarrow L_{q}}$. As in the proof of (4.5), one gets

$$
\left|\frac{\sin (2 n-1) \pi x}{\sin \pi x}\right| \leqslant 2 \min (n, 1 /|x|)
$$

and

$$
\sup _{1 \geqslant x \geqslant d} \frac{1}{x} \int_{0}^{x}\left|\frac{\sin (2 n-1) \pi t}{\sin \pi t}\right| d t \geqslant c \min (n, 1 / d)
$$

Hence, Theorem 4.2 implies

$$
\begin{aligned}
\sup _{\|f\|_{L_{p}([-\pi, \pi],|x| \gamma)}=1} & \left\|S_{n} \mid(f)\right\|_{L_{q}\left([-\pi, \pi],|x|^{-\beta}\right)} \\
& =\sup _{\|g\|_{L_{p}([-\pi, \pi],|x|-\alpha)}=1}\left\|\left|S_{n}\right|\left(g(\cdot)|\cdot|^{-\alpha}\right)\right\|_{L_{q}\left([-\pi, \pi],|x|^{-\beta}\right)} \\
& \asymp \sup _{2 a \geqslant d>0} \frac{1}{d^{1-\frac{1-\alpha}{p^{\prime}}-\frac{1-\beta}{q}}} \int_{-d}^{d}\left|\frac{\sin (2 n-1) \pi t}{\sin \pi t}\right| d t \\
& \asymp n^{1-\frac{1-\alpha}{p^{\prime}}-\frac{1-\beta}{q}}=n^{\frac{1+\gamma}{p}-\frac{1-\beta}{q}} .
\end{aligned}
$$

Thus, (4.3) and (4.4) are verified.

\section{Convolution operator in Lorentz spaces}

The following theorem provides the sufficient conditions for the convolution operator to be bounded in a Lorentz space.

Theorem 5.1. Let $1<p, q<\infty$ and let the measures $\mu$ and $\nu$ be on measurable sets $D$ and $\Omega$ from $\mathbb{R}^{n}$ respectively. Assume that a function $K(z)$ defined on $D-\Omega=\{z=x-y: x \in D, y \in \Omega\}$ satisfies the condition 
NET SPACES AND BOUNDEDNESS OF INTEGRAL OPERATORS

25

that there exists $\gamma>0$ such that

$$
\begin{aligned}
& \sup _{e \in M_{1}} \frac{1}{(\mu(e))^{1 / q^{\prime}-1 / \gamma p^{\prime}}}\left|\int_{e} K(x-y) d \mu_{y}\right| \leqslant B \quad \text { for a.e. } \quad x \in D, \\
& \sup _{w \in M_{2}} \frac{1}{(\nu(w))^{1 / p-\gamma / q}}\left|\int_{w} K(x-y) d \nu_{x}\right| \leqslant B \quad \text { for a.e. } \quad y \in \Omega,
\end{aligned}
$$

where $M_{1}=\{e \subset \Omega: 0<\mu(e)<\infty\}$ and $M_{2}=\{w \subset D: 0<\nu(w)<\infty\}$.

Then

$$
A f(y)=\int_{D} K(x-y) f(x) d \nu_{x}
$$

is bounded from $L_{p, h_{1}}(D, \nu)$ to $L_{q, h_{2}}(\Omega, \mu)$ with $1 \leqslant h_{1} \leqslant h_{2} \leqslant \infty$ and, moreover,

$$
\|A\|_{L_{p, h_{1}}(D, \nu) \rightarrow L_{q, h_{2}}(\Omega, \mu)} \leqslant C B
$$

where $C=C\left(p, q, h_{1}, h_{2}\right)$.

Proof. By Theorem 3.2 it is sufficient to prove

$$
I=\left(\int_{0}^{\infty}\left(\sup _{\mu(e) / \nu^{\gamma}(w)=s} F(e, w ; K)\right)^{r} \frac{d s}{s}\right)^{\frac{1}{r}} \leqslant C B,
$$

where

$$
F(e, \omega ; K)=\frac{1}{\nu(\omega)^{\frac{1}{p}}} \frac{1}{\mu(e)^{\frac{1}{q^{\prime}}}}\left|\int_{e} \int_{\omega} K(x-y) d \nu d \mu\right|,
$$

$\frac{1}{r}=\frac{1}{h_{2}}+1-\frac{1}{h_{1}} \leqslant 1$. To prove (5.1), we write $I^{r}=\int_{0}^{1}+\int_{1}^{\infty}=: I_{1}^{r}+I_{2}^{r}$ and estimate each term separately.

$$
\begin{aligned}
I_{1} & \leqslant\left(\int_{0}^{1}\left(\sup _{\mu(e) / \nu^{\gamma}(w)=s} \frac{1}{(\mu(e))^{1 / q^{\prime}}} \frac{1}{\nu(w)^{1 / p}}\left|\int_{e} \int_{w} K(x-y) d \nu d \mu\right|\right)^{r} \frac{d s}{s}\right)^{1 / r} \\
& \leqslant e s s \sup _{y \in \Omega} \sup _{\nu(w)>0} \frac{1}{\nu(w)^{1 / p-\gamma / q}} \\
& \left|\int_{w} K(x-y) d \nu\right|\left(\int_{0}^{1}\left(\sup _{\mu(e) / \nu^{\gamma}(w)=s} \frac{\mu(e)^{1 / q}}{\nu(w)^{\gamma / q}}\right)^{r} \frac{d s}{s}\right)^{1 / r} \\
& \leqslant B\left(\int_{0}^{1} s^{r / q} \frac{d s}{s}\right)^{1 / r}=(q / r)^{1 / r} B .
\end{aligned}
$$


Further,

$$
\begin{aligned}
I_{2} & \leqslant\left(\int_{1}^{\infty}\left(\sup _{\mu(e) / \nu^{\gamma}(w)=s} \frac{1}{(\mu(e))^{1 / q^{\prime}}} \frac{1}{\nu(w)^{1 / p}}\left|\int_{w} \int_{e} K(x-y) d \nu d \mu\right|\right)^{r} \frac{d s}{s}\right)^{\frac{1}{r}} \\
& \leqslant e s s \sup _{x \in D} \sup _{\mu(e)>0} \frac{1}{(\mu(e))^{1 / q^{\prime}-1 / \gamma p^{\prime}}} \\
& \left|\int_{e} K(x-y) d \mu\right|\left(\int_{1}^{\infty}\left(\sup _{\mu(e) / \nu^{\gamma}(w)=s} \frac{\nu(w)^{1 / p^{\prime}}}{\mu(e)^{1 / \gamma p^{\prime}}}\right)^{r} \frac{d s}{s}\right)^{1 / r} \\
& \leqslant\left(\gamma p^{\prime} / r\right)^{1 / r} B .
\end{aligned}
$$

Thus $I \leqslant C B$, which finishes the proof.

Now let us investigate the necessary conditions for boundedness of the convolution operator in Lorentz spaces. Let positive locally integrable functions $\nu(x)$ and $\mu(x)$ on $\mathbb{R}$ define the following measures

$$
\mu(e)=\int_{e} \mu(y) d y \quad \text { and } \quad \nu(w)=\int_{w} \nu(x) d x .
$$

Let $e=[a, b]$, then we denote by $[e]_{1},[e]_{2}$, and $[e]_{3}$ the following parts of $e:[e]_{1}=\left[a, a+\frac{b-a}{3}\right],[e]_{2}=\left[a+\frac{b-a}{3}, b-\frac{b-a}{3}\right]$, and $[e]_{3}=\left[b-\frac{b-a}{3}, b\right]$. For the function $\mu(x)>0$ on $\mathbb{R}$ we define the net

$$
M_{\mu}=\left\{[a, b]: \quad \frac{\mu(y)}{2} \leqslant \mu(x) \leqslant 2 \mu(y), \quad \forall x, y \in[a, b]\right\}
$$

Example 5.1. Suppose $\mu(x)=\frac{1}{|x|^{\beta}}, \beta>0$; then

$$
M_{\mu}=\left\{[a, b]: 0<a<b \leqslant 2^{\frac{1}{\beta}} a<\infty\right\} \bigcup\left\{[a, b]:-\infty<2^{\frac{1}{\beta}} b \leqslant a<b<0\right\} .
$$

Let $|e|$ be a length of an interval $e$. Note that for any $e \in M_{\mu}$ and $u \in\left[-\frac{|e|}{3}, \frac{|e|}{3}\right]$ one has

$$
\mu(e) \leqslant 6 \mu\left([e]_{2}+u\right),
$$


where $[e]_{2}+u=\left\{x+u: x \in[e]_{2}\right\}$. Indeed,

$$
\begin{aligned}
\mu(e) & =\int_{e} \mu(y) d y=\int_{[e]_{1}} \mu(y) d y+\int_{[e]_{2}} \mu(y) d y+\int_{[e]_{3}} \mu(y) d y \\
& =\int_{\left[e_{2}\right]+u}\left(\mu\left(y-\frac{|e|}{3}-u\right)+\mu(y-u)+\mu\left(y+\frac{|e|}{3}-u\right)\right) d y \\
& \leqslant 6 \int_{[e]_{2}+u} \mu(y) d y=6 \mu\left([e]_{2}+u\right) .
\end{aligned}
$$

Theorem 5.2. Let $1<p<q<\infty, 1 \leqslant h_{1} \leqslant h_{2} \leqslant \infty$, and let $\mu, \nu$ be defined by (5.2). Suppose $\nu$ and $\mu$ satisfy for some $\gamma>0$

$$
\begin{gathered}
\mu([0,|w| / 3]) \geqslant c_{1} \nu^{\gamma}(w) \quad \forall w \in M_{\nu}, \\
\nu^{\gamma}([0,|e| / 3]) \geqslant c_{2} \mu(e) \quad \forall e \in M_{\mu},
\end{gathered}
$$

and suppose that the convolution operator

$$
A g(y)=\int_{D} K(y-x) g(x) d \nu_{x}, \quad K(x) \geqslant 0,
$$

is bounded from $L_{p, h_{1}}(\mathbb{R}, \nu)$ into $L_{q, h_{2}}(\mathbb{R}, \mu)$; then

$$
\begin{aligned}
\sup _{e \in M_{\mu}} \sup _{0<y<\frac{|e|}{3}} & \frac{1}{\left(\mu\left([e]_{2}+y\right)\right)^{1 / q^{\prime}-1 / \gamma p^{\prime}}} \\
& \int_{[e]_{2}} K(z) \mu(z+y) d z \leqslant c\|A\|_{L_{p, h_{1}}(\mathbb{R}, \nu) \rightarrow L_{q, h_{2}}(\mathbb{R}, \mu),}, \\
\sup _{w \in M_{\nu}} \sup _{0<x<\frac{|w|}{3}} \frac{1}{\left(\nu\left([w]_{2}+x\right)\right)^{1 / p-\gamma / q}} & \int_{[w]_{2}} K(z) \nu(x+z) d z \leqslant c\|A\|_{L_{p, h_{1}}(\mathbb{R}, \nu) \rightarrow L_{q, h_{2}}(\mathbb{R}, \mu),},
\end{aligned}
$$

Conversely, suppose $M_{1}=\{e: 0<\mu(e)<\infty\}$ and $M_{2}=\{w: 0<\nu(w)<\infty\}$ and one of the following conditions holds true:

$$
\sup _{e \in M_{1}} \sup _{y \in \mathbb{R}} \frac{1}{(\mu(e+y))^{1 / q^{\prime}-1 / \gamma p^{\prime}}} \int_{e} K(z) \mu(y+z) d z \leqslant B
$$

or

$$
\sup _{w \in M_{2}} \sup _{x \in \mathbb{R}} \frac{1}{(\nu(x-w))^{1 / p-\gamma / q}} \int_{w} K(z) \nu(x-z) d z \leqslant B ;
$$

then the operator $A$ is bounded from $L_{p, h_{1}}(\mathbb{R}, \nu)$ into $L_{q, h_{2}}(\mathbb{R}, \mu)$ and

$$
\|A\|_{L_{p, h_{1}}(\mathbb{R}, \nu) \rightarrow L_{q, h_{2}}(\mathbb{R}, \mu)} \leqslant c B .
$$


Remark 5.1. The functions $\mu(y)=\frac{1}{|y|^{\beta}}, \beta \in[0,1)$ and $\nu(x)=\frac{1}{|x|^{\alpha}}, \alpha \in$ $[0,1)$ satisfy conditions $(5.4)$ with $\gamma=\frac{1-\beta}{1-\alpha}$.

Proof of Theorem. 5.2 By Corollary 3.1.1, we have

$$
\begin{aligned}
& \|A\|_{L_{p h_{1}}(\nu) \longrightarrow L_{q h_{2}}(\mu)} \geqslant \\
& \qquad \sup _{e \in M_{1}} \sup _{w \in M_{2}} \frac{1}{(\mu(e))^{1 / q^{\prime}}} \frac{1}{(\nu(w))^{1 / p}} \int_{e} \int_{w} K(y-x) \mu(y) \nu(x) d x d y .
\end{aligned}
$$

Let $e \in M_{\mu}$. Applying (5.3-5.4) and noting that for any $0<x \leqslant \frac{|e|}{3}$ one has $[e]_{2} \subset e-x$, we write

$$
\begin{aligned}
\|A\|_{L_{p h_{1}}(\nu)} & \longrightarrow L_{q h_{2}}(\mu) \\
& \geqslant \frac{c_{2}^{1 / p^{\prime}}}{\nu\left(\left[0, \frac{|e|}{3}\right]\right)} \int_{0}^{\frac{|e|}{3}} \nu(x) \frac{1}{(\mu(e))^{\frac{1}{q^{\prime}}-\frac{1}{\gamma p^{\prime}}}} \int_{e} K(y-x) \mu(y) d y d x \\
& \geqslant \frac{c_{2}^{1 / p^{\prime}}}{\mu\left(\left[0, \frac{|e|}{3}\right]\right)} \int_{0}^{\frac{|e|}{3}} \nu(x) \frac{1}{(\mu(e))^{\frac{1}{q^{\prime}}-\frac{1}{\gamma p^{\prime}}}} \int_{[e]_{2}} K(z) \mu(z+x) d z d x \\
& \geqslant \frac{c_{2}^{1 / p^{\prime}}}{2 \nu\left(\left[0, \frac{|e|}{3}\right]\right)} \int_{0}^{\frac{|e|}{3}} \nu(x)\left(\frac{1}{(\mu e)^{\frac{1}{q^{\prime}}-\frac{1}{\gamma p^{\prime}}}} \int_{[e]_{2}} K(z) \mu(z) d z\right) d x \\
& =\frac{c_{2}^{1 / p^{\prime}}}{2(\mu e)^{\frac{1}{q^{\prime}}-\frac{1}{\gamma p^{\prime}}}} \int_{[e]_{2}} K(z) \mu(z) d z \\
& \geqslant \frac{c_{2}^{1 / p^{\prime}}}{4 \cdot 6^{\frac{1}{q^{\prime}}-\frac{1}{\gamma p^{\prime}}}\left(\mu\left([e]_{2}+x\right)\right)^{\frac{1}{q^{\prime}}-\frac{1}{\gamma p^{\prime}}}} \int_{[e]_{2}} K(z) \mu(z+x) d z,
\end{aligned}
$$

where $x \in[0,|e| / 3]$. Therefore, taking into account arbitrary choice of $e \in$ $M_{\mu}$, we have

$$
\begin{aligned}
& \|A\|_{L_{p h_{1}}(\nu) \longrightarrow L_{q h_{2}}(\mu)} \geqslant \\
& \quad c \sup _{e \in M_{\mu}} \sup _{x \in\left[0, \frac{|e|}{3}\right]} \frac{1}{\left(\mu\left([e]_{2}+x\right)\right)^{\frac{1}{q^{\prime}}-\frac{1}{\gamma p^{\prime}}}} \int_{[w]_{2}} K(z) \mu(z+x) d z .
\end{aligned}
$$

Similarly,

$$
\begin{aligned}
& \|A\|_{L_{p h_{1}}(\nu) \longrightarrow L_{q h_{2}}(\mu)} \geqslant \\
& \quad c \sup _{w \in M_{\nu}} \sup _{y \in\left[0, \frac{|w|}{3}\right]} \frac{1}{\left(\nu\left(y-[w]_{2}\right)\right)^{\frac{1}{p}-\frac{\gamma}{q}}} \int_{[w]_{2}} K(z) \nu(y-z) d z .
\end{aligned}
$$


Let us now prove the second part of the theorem. By Theorem 3.2,

$$
\begin{aligned}
\|A\|_{L_{p h_{1}}(\nu) \longrightarrow L_{q h_{2}}(\mu)} & \leqslant c \sup _{e \in M_{1}} \sup _{x \in \mathbb{R}} \frac{1}{(\mu(e))^{\frac{1}{q^{\prime}}-\frac{1}{\gamma p^{\prime}}}} \int_{e} K(y-x) \mu(y) d y \\
& =c \sup _{x \in \mathbb{R}} \sup _{w \in M_{1}} \frac{1}{(\mu(e+x))^{\frac{1}{q^{\prime}}-\frac{1}{\gamma p^{\prime}}}} \int_{e} K(z) \mu(z+x) d z . \\
\|A\|_{L_{p h_{1}}(\nu) \longrightarrow L_{q h_{2}}(\mu)} & \leqslant c \sup _{w \in M_{2}} \sup _{y \in \mathbb{R}} \frac{1}{(\nu(y-w))^{\frac{1}{p}-\frac{\gamma}{q}}} \int_{w} K(z) \nu(y-z) d z .
\end{aligned}
$$

The proof is now complete.

\section{ACKNowledgements}

The authors are grateful to Fulvio Ricci for useful comments.

The research was supported by INTAS (05-1000008-815), the RFFI (Grants N 06-01-00268, N 08-01-00302), Russian Foundation for the Humanities (Grants N 08-06-00144a), the Leading Scientific Schools (Grant NSH2787.2008.1), and Scuola Normale Superiore. The paper was started when the authors were staying at the Centre de Recerca Matemática (Barcelona) in Spring 2006.

\section{REFERENCES}

[AH] K. F. Andersen, H. P. Heinig, Weighted norm inequalities for certain integral operators, SIAM J. Math. Anal., 14(4) (1983), 834-844.

[AS] K. F. Andersen, E. T. Sawyer, Weighted norm inequalities for the RiemannLiouville and Weyl fractional integral operators, Trans. Amer. Math. Soc., 308(2) (1988), 547-558.

[BH1] J. J. Benedetto, H. P. Heinig, Fourier transform inequalities with measure weights, Adv. Math., 96(2) (1992), 194-225.

[BH2] J. J. Benedetto, H. P. Heinig, Weighted Fourier inequalities: new proofs and generalizations, J. Fourier Anal. Appl., 9(1) (2003), 1-37.

[BS] C. Bennett and R. Sharpley, Interpolation of Operators, Academic Press, 1988.

[Bl] A. P. Blozinski, On a convolution theorem for $L_{p, q}$ spaces, Trans. Amer. Math. Soc. 164 (1972), 255-265.

[BS] B. Booton, Y. Sagher, Norm Inequalities for Certain Classes of Functions and their Fourier Transforms, To appear in Jour. of Math. Anal. Appl. Online: http://www.sciencedirect.com/science/journal/0022247X

[Bu] A. V. Bukhvalov, The integral representation of linear operators, (Russian) Zap. Nauchn. Semin. Leningr. Otd. Mat. Inst. Steklov 47 (1974), 5-14; translation in J. Soviet Math. 9 (1978), 129-137.

[CRS] M. J. Carro, J. A. Raposo, J. Soria, Recent Developments in the Theory of Lorentz Spaces and Weighted Inequalities, Mem. Amer. Math. Soc. 187, 2007.

[Ch] M. Christ, Weighted norm inequalities and Schur's lemma, Studia Math., 78 (1984), 309-319.

[CF] R. R. Coifman, C. Fefferman, Weighted norm inequalities for maximal functions and singular integrals, Studia Math., 51 (1974), 241-250. 
[DP] N. Dunford; B. Pettis, Linear operations on summable functions, Trans. Amer. Math. Soc., 47 (1940), 323-392.

[EKM] D. Edmunds, V. Kokilashvili, A. Meskhi, Bounded and compact integral operators, Mathematics and its Applications, 543. Kluwer Academic Publishers, 2002.

[EO] D. Edmunds, B. Opic, Equivalent quasi-norms on Lorentz spaces, Proc. Amer. Math. Soc., 131(3) (2003), 745-754.

[Ga] E. Gagliardo, On integral transformations with positive kernel, Proc. Am. Math. Soc., 16, 429-434 (1965).

[GR] J. García-Cuerva, J. Rubio de Francia, Weighted norm inequalities and related topics, North-Holland, 1985.

[H1] E. Hernández, Weighted inequalities through factorization, Publ. Mat., 35(1) (1991), 141-153.

[H2] E. Hernández, Factorization and extrapolation of pairs of weights, Stud. Math., 95(2) (1989), 179-193.

[Ja] B. Jawerth, Weighted inequalities for maximal operators: Linearization, localization and factorization, Amer. J. Math., 108 (1986), 361-414.

[KA] L. V. Kantorovich; G. P. Akilov, Functional analysis, Second edition. Pergamon Press, Oxford-Elmsford, N.Y., 1982.

[KV] L. V. Kantorovich, B. Z. Vulikh, Sur la representation des operations lineaires, (French) Compositio Math., 5, 119-165 (1937).

[Ke] R. A. Kerman, Convolution theorems with weights, Trans. Amer. Math. Soc., 280(1) (1983), 207-219.

[KN] A. G. Kostyuchenko, E.D. Nursultanov, On integral operators in $L_{p}$-spaces, Fundam. Prikl. Mat. 5(2) (1999), 475-491.

[KP] A. Kufner, L.-E. Persson, Weighted inequalities of Hardy type, River Edge, NJ, 2003.

[MT] G. Mastroianni, V. Totik, Weighted polynomial inequalities with doubling and $A_{\infty}$ weights, Constr. Approx., 16(1) (2000), 37-71.

[Mu] B. Muckenhoupt, Hardy's inequality with weights, Studia Math., 44 (1972), 31-38.

[Nu] E. D. Nursultanov, Net spaces and inequalities of Hardy-Littlewood type, Sb. Math. 189(3) (1998) 399-419; translation from Mat. Sb. 189(3), (1998), 83-102.

[ON] R. O'Neil, Convolution operators and $L(p, q)$ spaces, Duke Math. J., 30 (1963), $129-142$.

[Sa] Y. Sagher, Integrability conditions for the Fourier transform, J. Math. Anal. Appl., 54(1) (1976), 151-156.

[SW] E. Stein, G. Weiss, Introduction to Fourier analysis on Euclidean spaces, Princeton, N.J., 1971.

[Ya] L. Y. H. Yap, Some remarks on convolution operators and $l(p, q)$ spaces, Duke Math J., 36 (1969), 647-658. 
E. Nursultanov

Kazakh Branch of Moscow State University

Munatpasova, 7

010010Astana KaZAKHStan

E-mail address: er-nurs@yandex.ru

S. TikHONOV

Scuola Normale Superiore

Piazza dei Cavalieri, 7

56126 PISA ItAly

E-mail address: s.tikhonov@sns.it 